CAHIERS DE

NARRATOLOGIE

\section{Cahiers de Narratologie}

Analyse et théorie narratives

Littérature et sciences

\title{
Mémoire et imagination chez les aliénistes esquiroliens et dans la troisième version de la Tentation de saint-Antoine de Flaubert : d'un savoir l'autre
}

Jean-Louis Cabanès

\section{CpenEdition}

Journals

Édition électronique

URL : http://journals.openedition.org/narratologie/5970

DOI : 10.4000/narratologie.5970

ISSN : 1765-307X

Éditeur

LIRCES

Référence électronique

Jean-Louis Cabanès, « Mémoire et imagination chez les aliénistes esquiroliens et dans la troisième version de la Tentation de saint-Antoine de Flaubert : d'un savoir l'autre », Cahiers de Narratologie [En ligne], 18 | 2010, mis en ligne le 05 janvier 2011, consulté le 19 avril 2019. URL : http:// journals.openedition.org/narratologie/5970 ; DOI : 10.4000/narratologie.5970

Ce document a été généré automatiquement le 19 avril 2019

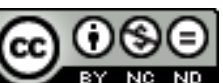

Cahiers de Narratologie - Analyse et théorie narratives est mis à disposition selon les termes de la licence Creative Commons Attribution - Pas d'Utilisation Commerciale - Pas de Modification 4.0 International. 


\title{
Mémoire et imagination chez les aliénistes esquiroliens et dans la troisième version de la Tentation de saint-Antoine de Flaubert : d'un savoir l'autre
}

\author{
Jean-Louis Cabanès
}

L'aliénisme, sous la Monarchie de Juillet et sous le Second Empire, ne se cantonne pas dans la sphère qui, par définition, est la sienne : la médecine de l'esprit. La considération des symptômes, le désir de les expliquer, la volonté de délimiter un territoire que l'on ne saurait confondre avec celui de la pathologie interne, engagent une réflexion sur les passions, sur la morale et plus largement sur les facultés: mémoire, imagination, attention. La médecine mentale se trouve ainsi au carrefour de plusieurs champs, elle ne peut ignorer l'anatomo-pathologie avec qui elle entre parfois en concurrence, elle rencontre la philosophie sur laquelle elle veut gager une psychologie. Conforté par la création de l'institution asilaire, reconnu comme expert par la justice au nom de la loi de 1838 sur l'internement arbitraire, l'aliéniste devient le spécialiste de la science de l'homme et, comme tel, il étend ses investigations dans plusieurs directions. Il s'interroge sur les formes de la déraison dans l'histoire, et pratique à sa manière "la médecine rétrospective ", il réexamine la question ancienne, sans cesse débattue depuis l'Antiquité, des relations qu'entretiennent folie et création, ce qui le conduit à s'aventurer dans les débats esthétiques. Jugeant les hommes, il juge aussi les œuvres en se demandant ce qu'il faut penser de l'inspiration, de l'enthousiasme, de l'épanchement du rêve dans la vie, de la prégnance chez l'artiste de la vie imaginative, de la fixation de l'attention sur le monde des idées. Les discussions sur le rêve et la folie, sur la nature des hallucinations - controverses auxquelles participe un historien comme Alfred Maury - conduisent les aliénistes à s'interroger sur le rôle des grands hommes dans l'Histoire, ou bien encore à scruter la psychologie de la création artistique. Les discours qui en résultent délimitent ce qu'on pourrait appeler un cadre épistémique dont on voudrait montrer qu'il fournit un 
cadre rhétorique à Flaubert, dans la troisième version de la Tentation de saint-Antoine. On se fondera plus particulièrement sur l'épisode des sept péchés capitaux. Dans le prolongement de ces analyses, on considérera, à la lumière des thèses défendues par l'aliéniste Brierre de Boismont, la coupure instaurée par l'écrivain, dans ses échanges épistolaires avec Taine, entre hallucination artistique et hallucination pathologique. Ce cheminement conduira certes à envisager des questions d'esthétique et de psychologie, peut-être aussi de poétique, il permettra de saisir également comment l'on glisse avec Flaubert d'un savoir à un autre alors que l'écrivain semble demeurer en accord parfait avec l'aliénisme esquirolien ou avec les thèses défendues par Alfred Maury. Notre propos est donc double. Il invite, d'une part, à s'interroger sur la métamorphose des médecins en critiques littéraires ou en esthètes, et d'autre part sur la manière dont la littérature infléchit un discours dont cependant elle s'inspire.

2 Esquirol et ses disciples, Lélut, Moreau de Tours, Baillarger, ont en commun d'envisager l'hallucination pathologique comme un exercice involontaire des facultés. Certes, l'étiologie des phénomènes hallucinatoires diverge d'un aliéniste à un autre, tout comme les frontières qu'ils croient bon de tracer entre le normal et le pathologique. Mais tous s'accordent, à l'exception peut-être du seul Leuret dans ses Fragments psychologiques, pour envisager les hallucinations qui parasitent le sujet comme des images déformées ayant pour soubassement des traces mémorielles plus ou moins lointaines. Esquirol, dans son traité Des maladies mentales, estimait que les « prétendues sensations des aliénés sont des images, des idées produites par la mémoire, associées par l'imagination et personnifiées par l'habitude. L'homme donne corps au produit de son entendement. Il rêve tout éveillé [...] Les rêves comme les hallucinations, reproduisent toujours des sensations, les idées anciennes ${ }^{1} »$. Tout ici mériterait une analyse approfondie. On pourrait s'interroger, en effet, sur la relation des idées aux images, sur le passage des unes aux autres (Lélut, Brierre de Boismont, Baillarger, l'historien Alfred Maury, Taine, soulèvent ce type de questions, nous y reviendrons); on pourrait aussi se demander si l'imagination, telle que la conçoit Esquirol, ne se réduit pas à une combinatoire d'images mentales; on constate également qu'il tend à faire du rêve le paradigme de la folie : Moreau de Tours publiera chez Louis Martinet, en 1855, un article intitulé De l'identité de l'état de rêve et de la folie et Alfred Maury adoptera en partie sur ce point les thèses de cet aliéniste dans son grand livre sur Le Sommeil et les Rêves publié en 1861. Cette citation d'Esquirol figure donc une sorte de carrefour, d'échangeur remarquable dont l'intérêt pour nous tient précisément aux liens que ce discours instaure entre l'exercice involontaire des facultés et l'hallucinatoire, entre le sensoriel et l'idéel, entre le surgissement de matériaux mnésiques et la dynamique de l'imagination, faculté qu'une psychologie associationniste réduit à combiner des images. L'halluciné serait victime d'une dynamique mortifère, celle de l'enchaînement de ce qu'on n'appelle pas encore des fantasmes. Il serait à proprement parler « aliéné » par des images. Il y aurait ainsi, selon Esquirol, une rhétorique déréglée de l'imaginaire qui se traduirait tantôt en termes de fixité (la personnification par l'habitude des images mentales), tantôt par une cinétique délétère: les images se bousculeraient, conglomérées ou associées par analogie ou contiguïté.

3 Explorons d'abord une première piste en rappelant que la proximité de l'hallucinatoire et du souvenir tient d'une vulgate, tout comme la mise en relation étroite de la mémoire et de l'imagination. Baillarger estimait ainsi, dans un article de 1846, qu'il ne saurait y avoir hallucination sans l'exercice involontaire de la mémoire et de l'imagination ${ }^{2}$, Louis Peisse, lors des débats qui se déroulèrent, en 1855-1856, dans le cadre de la Société des études 
médico-psychologiques, reprenait la même antienne : «l'hallucination, ce phénomène en apparence si étrange, n'est que la répétition des opérations de la mémoire et de l'imagination, s'exerçant spontanément et involontairement avec un degré insolite d'énergie ${ }^{3}$ ", tandis que Brierre de Boismont affirmait que les hallucinations sont

la revivification de ces milliards d'images, des sonorités, d'impressions tactiles, etc. qui ne peuvent exister dans notre cerveau qu'à condition de perdre leurs signes sensibles, et qu'on serait tenté de comparer au mystère de la résurrection des corps, lorsqu'ils reparaissent avec les attributs de la sensation ${ }^{4}$.

4 Cette part de la mémoire, selon Maury, est plus grande dans notre activité mentale que nous le croyons ordinairement, parce que le «souvenir est inscient dans une foule de faits; tel paraît être notamment le caractère des souvenirs d'enfance ${ }^{5} »$. Les hallucinations, selon Brierre, les rêves et les hallucinations hypnagogiques, selon Maury, ont donc un aspect lazaréen, elles ressuscitent, certes de manière déformée, ce qui serait peut-être demeuré de l'ordre de l'insu.

On peut alors se demander comment les aliénistes envisagent les autres productions de la mémoire et de l'imagination, et notamment celles qui résultent de l'imagination créatrice de l'artiste. Ces sujets ont été abordés remarquablement par Tony James et par Juan Rigoli ${ }^{6}$. On se bornera ici à quelques remarques en rappelant ce qui oppose Brierre de Boismont à la plupart de ses confrères. Ami de Vigny, dont il soigna la femme, cet aliéniste catholique, qui veilla autant qu'il le put sur le malheureux Charles Lassailly, estimait qu'il fallait considérer les représentations mentales de manière unitaire, en distinguant néanmoins les hallucinations compatibles avec la santé, qu'il appelait physiologiques, des hallucinations pathologiques. La première thèse, entendons l'unicité des représentations mentales, implique un glissement possible de l'idée au sensible, de la sensation à l'idée. Cette connexion supposée tient, comme l'on a vu précédemment, d'un lieu commun, et nombre d'aliénistes, de médecins, sont prêts à la proclamer pour des raisons opposées. Lélut, héritier des idéologues, voit dans l'hallucination une sorte de régression esthésique, un processus involutif, un retour de l'idée à son origine sensible. Le docteur Louis Peisse, qui penche vers l'éclectisme, estime que

la mémoire, la conception, l'illusion, l'hallucination et les diverses formes d'état psychique ont - en les dégageant de toutes les diverses formes, de toutes les circonstances accidentelles - pour fonds commun et identique, pour élément essentiel, le phénomène normal de la perception sensorielle, de la sensation ${ }^{7}$

6 La conversion de la sensation en représentation, en idée, en conception joue également un rôle fondamental dans la psychologie de Taine qui postule, d'une part que nous n'appréhendons le réel que sous forme d'images et qu'il existe, d'autre part, une similitude entre la «conception (cum-capere, la chose devenue interne), la « représentation (rursus praesens, la chose présente de nouveau, quoiqu'en fait absente), l'« idée (eidos, la figure, le semblant, l'apparence de la chose, au lieu de la chose elle-même $8 »$. On pourrait prolonger ces constats en rappelant l'identité de fait, pour Taine, de la perception, cette hallucination vraie, et de l'hallucination. Pour autant, il ne s'agit pas de mettre sur le même plan les thèses soutenues par le philosophe-historien et par Brierre, mais bien de faire surgir un cadre épistémique qui autorise non plus seulement la prise en compte dans l'hallucinatoire du mémoriel, mais la possibilité d'y découvrir des idéesimages pour parler le langage de Maury, ou des idées-sensations, pour reprendre le vocabulaire de Lélut. On laissera de côté ici le partage opéré par Baillarger entre hallucinations psychiques et hallucinations psycho-sensorielles bien qu'il fasse sens à l'intérieur du cadre que l'on vient de délimiter. 
7 Les trajets conceptuellement et psychologiquement flous qui permettent d'aller des idées aux sensations, aux images, aux hallucinations, légitiment le parcours antithétique, sur le plan idéologique, de Lélut et de Brierre. Frères ennemis, ils participent du même paradigme. En effet, si l'on peut glisser de l'idée au sensible sous la forme d'une hallucination pathologique, rien d'étonnant à ce que de grands esprits soient victimes de semblables désordres - ce serait le cas de Pascal ou de Socrate, dont Lélut s'est fait, dans ses essais, le médecin rétrospectif. Ils auraient été assiégés par des hallucinations que l'on pourrait dire centrifuges : "Elles seraient le retour des idées à leur point de départ ", déclare Lélut qui précise «j'entends des idées sensibles, de celles qui deviennent immédiatement des sensations, qui ont en un mot leur origine dans les sentimentssensations de Laromiguière ${ }^{9}$. Cette machinerie régressive a pour effet de faire tomber les extatiques et les visionnaires à la clinique.

Dans un cheminement symétrique, Brierre de Boismont, précisément parce que l'on peut passer graduellement de l'idée à la sensation ou de l'idée à l'hallucination, tente de prouver, à l'inverse de Lélut, que les fondateurs de religions, les mystiques, les grands artistes ont tout naturellement des visions. Ce qui était signe pathologique, infirmité se retourne en signe d'élection. Si la représentation mentale et l'hallucination se ressemblent, l'homme qui pense, qui concentre son attention, peut voir se corporifier ses idées. Voici d'une part que la mystique se sauve de toute déraison, elle est le stade ultime d'une méditation, tandis que, d'autre part, la création artistique s'émancipe de la folie: «Les poètes, les peintres, les sculpteurs que le génie a effleurés de son aile, ont aperçu devant eux, après des méditations prolongées, la forme de l'idéal qu'ils avaient rêvé. Leur histoire atteste que cette forme était visible aux yeux de l'esprit, pour nous servir de l'expression si pittoresque de Shakespeare, et souvent même aux yeux de leur corps[...] ou du moins s'offrait à eux avec les caractères d'extrémité de l'hallucination. Il y a plus, c'est que nous ne croyons pas qu'il y ait de créations immortelles, sans cette matérialisation de l'idéal ${ }^{10}$." L'hallucination physiologique, mais nous serions tentés de dire aussi l'imagination qui la gouverne, impliquent une incarnation de l'idée qui devient image. Et l'image se fait chair pour mieux informer une action ou une œuvre.

9 Parallèlement, comme l'on s'en doute, Brierre récuse l'idée que le rêve puisse fournir le modèle paradigmatique de la folie, en s'opposant radicalement, sur ce point, à Moreau de Tours. Pour cet aliéniste, on le sait, le rêve et l'hallucination ressortissent à une commune excitation du cerveau, et l'un explique l'autre. On comprend alors pourquoi Aurélia a pu être considéré par Moreau comme un merveilleux document, puisque précisément la folie, et l'on ne saurait imaginer, estime-t-il, meilleure formule, c'est « l'épanchement du songe dans la vie réelle ${ }^{11} »$. Il est pour le moins significatif que l'opuscule de Moreau, De l'identité de l'état de rêve et de la folie, ait paru en 1855, l'année de la mort de Nerval et après que l'on eut publié ce récit inachevé, Aurélia.

10 Brierre s'empare lui aussi du récit nervalien comme d'un document quasi biographique, mais pour mieux différencier le rêve de la folie. «Il existe, déclare-t-il, d'ailleurs un grand livre qui a pour titre: Le Rêve et la vie, trouvé sur un écrivain de grand talent, d'une imagination prodigieuse, qui connaissait bien la folie, pour en avoir été visité, et qui en parlait d'une manière très remarquable. Dans ce livre de l'infortuné Gérard de Nerval, il y a des rêves, et il les distingue avec beaucoup de soin de la rêverie, qui sont si bien suivis, où les raisonnements, quoique planant dans les espaces infinis, sont d'un tel intérêt, si bien liés, qu'il est impossible d'y constater l'absence d'idées intermédiaires. Aussi répétons-nous que si le rêve, comparé à la folie, peut fournir matière à d'intéressants 
parallèles, il ne saurait lui être assimilé1 ${ }^{12}$.» On créditera Brierre d'une lecture fine d' Aurélia, d'une écoute attentive du texte de Nerval, car précisément l'écrivain a distingué rêve et folie, tout au moins récit de rêve et séquence à proprement parler hallucinatoire, et il n'a eu de cesse, d'en appeler contre le savoir médical à un savoir autre, tout en faisant la part de ce que les aliénistes prétendent connaître de la folie.

11 Certes, Brierre ne lit pas le texte nervalien pour lui-même, son approche est préorientée par une démonstration. Il s'agit pour l'aliéniste de distinguer soigneusement ce qui est pathologique et ce qui ne l'est point, pour mieux exalter la puissance de l'esprit, la dimension spirituelle de la création artistique, quitte à faire l'éloge enthousiaste de la rêverie en se référant à une lettre que Vigny lui aurait envoyée ${ }^{13}$. Qu'est-ce que rêver éveillé, sinon se donner à soi-même, volontairement, des images en mettant en branle, de manière active, son imagination? L'hallucination physiologique figure donc, si l'on en croit cet aliéniste, «le couronnement d'une méditation». Celle-ci suit la pente de la rêverie, explore les promontoires du songe. Le rêve éveillé, dirigé, est connaissance, il ne saurait être dissocié de l'intuition. Le propre des grands hommes, comme le dit Brierre en s'inspirant de Carlyle, c'est qu'ils communiquent, par le truchement des hallucinations physiologiques, avec le mystérieux infini ${ }^{14}$. Ces hallucinations figurent donc la passerelle qui conduit le sujet méditatif et rêveur vers une extase de savoir, et peut-être faut-il les considérer comme cette extase même. À la suite de Victor Cousin, Brierre isolait trois modes de la connaissance, la déduction, l'induction et l'intuition ${ }^{15}$. Les méditations des grands hommes seraient des intuitions devenues actes, non seulement dans leurs écrits ou dans leurs œuvres, mais au moment même où leurs visions se corporifient. On peut donc apercevoir dans les trois versions du traité Des Hallucinations $(1845,1852,1862)$ tout à la fois une psychologie de la voyance, de la rêverie, de l'imagination créatrice, une réflexion sur la valeur cognitive de l'imagination et bien évidemment une esthétique informée par la littérature romantique.

12 A-t-on prêté à Brierre plus que la lecture de son grand livre nous donne en réalité ? On a tout au moins négligé des tensions et peut-être des inconséquences qu'on n'ose baptiser des apories. Comment, en effet, concilier l'exaltation de l'imagination créatrice et la thèse selon laquelle les hallucinations, quelle que soit leur nature, sont toujours des réminiscences? À la suite de Philarète Chasles et de Théophile Gautier ${ }^{16}$, Brierre découvrait en Balzac un visionnaire, un voyant. Mais cette voyance, si l'on scrute les termes utilisés par l'aliéniste, est liée à l'idée de reproduction, de représentation, de mémoire. On n'est plus dans le domaine de l'idéel, ni dans celui de la rêverie. Ainsi, les visions balzaciennes seraient quasi photographiques. Elles concurrencent le réel. Certes, comme le dit Taine, cité par Brierre de Boismont, chez l'auteur de La Comédie humaine, "l'intensité de l'hallucination est la source de la vérité ${ }^{17}$ ", toutefois, ce n'est ni la notion d'intensité, fort intéressante car elle implique une esthétique, ni celle de vérité qui importent à l'aliéniste, mais la capacité du romancier à faire reparaitre à volonté les impressions emmagasinées. Cette faculté lui aurait permis de retracer les objets et les personnes qui auraient posé devant lui. Il en résulterait ces «admirables descriptions d'intérieur » et les «nombreux types qui jouent un rôle plus ou moins important dans La Comédie humaine ${ }^{18}$. ». Nous sommes alors fort loin de l'imagination telle que la conçoit Baudelaire dans le prolongement de Coleridge ou des romantiques allemands.

13 Mais voici qu'un autre discours semble informer l'esthétique de Brierre de Boismont. Il reprend pour ses démonstrations les thèses traditionnelles sur la beauté comme cosa mentale. L'aliéniste, au cours du débat qui se déroula à la Société des Études médico- 
psychologiques, avait cité en effet, à ce propos, une lettre bien connue de Raphaël à Balthazar Castiglione. Il s'y réfère encore dans son traité sur les hallucinations ${ }^{19}$. Le peintre y soulignait que, faute de trouver dans la réalité des modèles qui correspondissent à son idéal, il avait dû aller les chercher dans son monde intérieur. En réalité, qu'il s'agisse de la voyance photographique balzacienne, de l'œil intérieur du peintre, de l'audition interne du musicien, voire de la rêverie romantique, ce qui importe à Brierre, ce n'est pas tant de proposer une conception cohérente de l'imagination créatrice que de faire apparaître l'existence, chez les créateurs et les penseurs, d'une représentation interne qui se situe à l'intersection du sensible, de l'idéel, de l'image, et qui se présenterait toujours comme une hallucination. Cette représentation est en même temps connaissance, elle est intuition, dépassement de la raison analytique. En superposant ainsi des conceptions en partie opposées de l'imagination pour mieux sauvegarder l'idée platonicienne d'œil de l'esprit, d'œil intérieur, sans pour autant abandonner une approche psychologique, Brierre se soustrayait à cette réduction pathologique dont les aliénistes de son temps étaient coutumiers lorsqu'ils découvraient dans tout génie, dans tout extatique, dans tout enthousiaste des stigmates de déraison. Tandis que Lélut prolongeait les idéologues, tandis qu'Esquirol réactualisait une psychologie associationniste que l'on retrouve encore chez Maury ou chez Brierre, celuici, inspiré par le romantisme, se mettait à l'écoute de l'éclectisme cousinien, pour faire la part de l'imagination et de l'intuition. Victor Hugo dans les Quatre vents de l'esprit s'exclamait: "Socrate est fou: lisez Lélut qui le confond", et Baudelaire, dans "Assommons les pauvres ", déclarait en écho: «Puisque Socrate avait son bon démon, pourquoi n'aurais-je pas l'honneur comme Socrate, d'avoir mon brevet de folie, signé du subtil Lélut et du bien avisé Baillarger »? Les sarcasmes baudelairiens ne furent jamais dirigés contre Brierre qui, par ailleurs, cita élogieusement Les Paradis artificiels dans la troisième version de son grand traité. Notre aliéniste s'était fondé sur la littérature romantique, sur l'idée de surnaturalisme, pour déjouer ce qui ressortit encore à la philosophie sensualiste chez Lélut et chez Esquirol, ou pour mettre en cause l'organicisme de Moreau de Tours.

Si l'on a voulu, dans un premier temps, s'interroger sur la relation qu'entretiennent mémoire et imagination, en prenant pour fil directeur ce qui fait de Brierre un disciple d'Esquirol tout à la fois exemplaire et dissident, on a tracé, dans un même élan, un cadre épistémique qui deviendra pour Flaubert, dans la troisième version de La Tentation de saint Antoine, un cadre rhétorique. L'organisation des séquences des quatre premières sections de La Tentation, à savoir le monologue initial d'Antoine, les tableaux des sept péchés capitaux, l'apparition de la reine de Saba, le dialogue avec Hilarion, est, en effet, en harmonie avec l'idée que les aliénistes esquiroliens se font des hallucinations dans les rapports que celles-ci entretiennent avec les souvenirs, avec le rêve, avec les impressions sensibles devenues traces mémorielles. Toutefois, en scrutant plus attentivement la manière dont se trouvent convoqués les sept péchés capitaux, en analysant leur ordre de présentation, les tableaux que leur succession présuppose, on entend également montrer que Flaubert invente sur le plan rhétorique et poétique, en faisant dériver l'aliénisme esquirolien vers des domaines autres, celui que le freudisme explorera, mais aussi celui d'une morale anti-ascétique qu'informe en partie Spinoza. Avant d'analyser le texte de la Tentation, il nous faut faire encore un détour en complétant, si l'on peut dire Esquirol ou Brierre par Alfred Maury, parce que celui-ci, dans la production des rêves et des hallucinations, ne néglige pas la part des besoins et de penchants, des désirs censurés, tout en se centrant sur la manière dont les images se combinent et se conglomèrent. 

Moreau de Tours nous semble être allé dans ce sens - est marqué par le rôle qu'il accorde, dans les épisodes oniriques, à l'éloquence des besoins et des désirs : elle est d'autant plus forte que, dans les rêves, la censure ne s'exerce plus.

Dans les songes, déclare-t-il, je me laisse aller aux accès les plus violents de la colère, aux désirs les plus effrénés, et quand je m'éveille, j'ai presque honte de ces crimes imaginaires. Évidemment, les visions qui se déroulent dans ma pensée et qui constituent le rêve me sont suggérées par les incitations que je ressens et que ma volonté absente ne cherche pas à refouler ${ }^{20}$.

Les visions sont ainsi des satisfactions imaginaires. Maury donne l'exemple d'une hallucination hypnagogique qui, alors qu'il était sous l'empire de la faim, lui fit voir « une assiette et un mets qu'y prenait une main armée d'une fourchette. Endormi, quelques minutes après, déclare-t-il, je me trouvai à une table bien servie ${ }^{21}$ ».

La psychologie associationniste de Maury veut enfin que les hallucinations ou les rêves combinent plusieurs images, le passage des unes aux autres ou leur conglomération s'effectuent par des liens qui n'ont rien de rationnel et que nous saisissons d'autant moins que les idées sont devenues des images. Ces liens sont souvent des associations de mots, de signes. Si le rêve tient d'une combinatoire de visions qui s'enchaînent entre elles par un élément médian, l'hypothèse des hallucinations psychiques, chère à Baillarger, présuppose aussi la possibilité d'un fractionnement de la personnalité. Il arrive, également dans le rêve, déclare Maury, que nous attribuions

à des personnages différents, des pensées, des paroles qui ne sont autres que les nôtres. Dans un des rêves les plus clairs, les plus raisonnables que j'ai eus, je soutenais, avec un interlocuteur, une discussion sur l'immortalité de l'âme, et tous deux nous faisions valoir des arguments opposés, qui n'étaient autres que les objections que je me faisais à moi-même ${ }^{22}$.

Nous avons relevé un peu longuement ces quelques citations, car elles permettent de donner forme concrète à ce cadre notionnel dont nous voudrions montrer maintenant qu'il devient dans la troisième version de La Tentation un cadre rhétorique. L'habileté de l'écrivain consiste, en effet, à susciter, dans la section I, sous forme de monologue, une anamnèse. La parole du souvenir, en ce commencement de La Tentation, développe des images et des paysages qui vont s'imposer à nouveau, mais déformés, transformés, dans les rêves et les hallucinations d'Antoine. Prenons, par exemple, l'évocation d'Alexandrie dans la section I. Antoine se rappelle le Paneum où il se promenait avec Didyme et d'où l'on découvre le Phare et la haute mer. On retrouvera par la suite ce paysage dans la séquence des péchés capitaux. Quant au défilé des hérésies, ou à la présence d'un gymonosophiste, dans la section IV, ils sont subordonnés au séjour alexandrin de l'ermite : « leurs discours me reviennent quelquefois dans la mémoire ${ }^{23}$. » Il est également aisé de relier, dans la section II, la dérive onirique d'Antoine qui paresse dans un lit devenu barque, ou bien encore le " bruit de grelot, de tambours et de chanteurs ", produit par des "gens qui s'en vont à Canope ${ }^{24}$ ", à ce passage de la section I : «Les marchands d'Alexandrie naviguent les jours de fête sur la rivière de Canope, et boivent du vin dans des calices de lotus, au bruit des tambourins qui font trembler les cavernes le long du bord !25 " On pourrait multiplier longuement les exemples, la biographie d'Antoine par lui-même, au tout commencement de l'ouvrage, qu'il s'agisse de l'évocation d'Alexandrie, de celle de Byzance, figure une matrice mémorielle d'où semblent découler toutes les autres séquences jusqu'au défilé des dieux, tournant essentiel où, à la mémoire d'Antoine 
se substitue la mémoire de l'humanité, tout au moins celle qu'elle pourrait conserver des mythes, des « fictions » qu'elle a inventés.

C'est encore en conformité avec les exemples donnés par Maury dans Le Sommeil et les rêves que les lectures de l'ermite se prolongent sous forme d'hallucinations. Sur ce point, on sera bref car tout a déjà été dit. La lecture des passages de l'Ancien Testament se focalise sur trois personnages. La figure de Nabuchodonosor cristallisera par la suite, dans la section II, le péché d'envie, les trésors d'Ézéchias reparaîtront dans la tentation de l'avarice, la reine de Saba sera bien évidemment associée à la luxure. Quant au passage des Actes des apôtres relatifs aux massacres des juifs, il viendra alimenter la tentation de la colère. Toutefois, entre les séquences hallucinatoires et les lectures d'Antoine, il faut faire intervenir un chaînon intermédiaire. Toute citation du texte biblique se prolonge en rêverie ou bien en discours avec soi-même tenu en pleine conscience. Le trésor d'Ézéchias amène Antoine à s'exclamer : «Je me figure... qu'on voyait entassés jusqu'au plafond des pierres fines, des diamants, des dariques ${ }^{26}$.» Quant aux évocations de la reine de Saba et de Nabuchodonosor, dans la section I, elles provoquent des doutes, des objections, elles suscitent une parole critique, ou tout au moins une discussion avec $\operatorname{soi}^{27}$ qui se répercutera par la suite dans les sections III, IV. Hilarion, présenté dans la section I comme un disciple pensif et interrogateur ${ }^{28}$ deviendra dans la section III un double particulièrement interrogant qui « sait tout » de l'ermite ${ }^{29}$. En réalité, il figure une sorte de moi partiel qui s'autonomise sous l'effet d'une hallucination. Maury déclarait que, dans ses rêves, il lui arrivait de s'adresser, par interlocuteur interposé, des objections à lui-même. Hilarion est la projection raisonneuse d'une altérité intérieure qui ferait vaciller les certitudes du sujet s'il lui donnait libre cours.

20 Comme on le voit, la première partie de la Tentation s'organise selon une double disposition. Soit l'ermite se souvient, et le mouvement rétrospectif de l'anamnèse amorce la remontée dans le présent d'un matériau déjà travaillé par l'imagination - le souvenir est, en effet, un matériau second, l'analogon en partie fantasmé d'un sédiment mémoriel -, soit Antoine, dans le présent du monologue, lit un texte biblique, mais les rêveries qui en résultent viennent doubler le texte cité sous forme de discours ou d'image mentale. Le travail du rêve ou les métamorphoses que les hallucinations font subir, par la suite, aux souvenirs et aux lectures sont déjà amorcés au sein de cette première partie parce qu'elle nous met d'emblée en contact avec un sujet discourant, imaginant, glosant, se retournant sur lui-même ou questionnant son propre savoir. En ce sens, la section I, est déjà le lieu où s'effectue la duplication subjectivée d'un matériau livresque ou mémoriel que les séquences suivantes vont encore dupliquer et transformer dans la dynamique même de la reproduction.

21 Toutefois, comme le dirait Maury, les images oniriques ou hallucinatoires sont subordonnées à nos penchants, à nos besoins. Ceux-ci se manifestent, sous l'effet de l'ascétisme, dès la section I. Le monologue d'Antoine y fait entendre l'éloquence de la chair frustrée ou bien celle des appétits et des passions. Tour à tour la gourmandise («Ah! de la chair rouge... une grappe de raisin qu'on mord $\left.{ }^{30} ! »\right)$, la luxure (on renvoie à l'évocation d'Ammonaria), la colère ("Voilà que je plonge dans des idées de meurtre et de sang ${ }^{31}$ !), l'orgueil (Antoine glorifie son activité érémitique, exalte sa notoriété), l'envie (il rêve d'être autre, jalouse les pères du concile de Nicée), l'avarice (nous avons mentionné les rêveries sur le trésor d'Ézéchias), et peut-être la paresse (les plaintes incessantes de l'ermite sur son long martyr sont autant d'appel au repos) surgissent dans le discours de l'anamnèse sous forme de tentations. L'on voit ainsi se configurer, dès la 
première section, des pulsions, des appétits qui vont se développer, se regrouper sous le signe traditionnel des sept péchés capitaux dans la section II.

Le monologue initial de l'ermite figure donc, conformément aux relations que la psychiatrie post-esquirolienne instaure entre mémoire, rêve, hallucination, besoin, la strate consciente (mais déjà néanmoins fantasmatique) d'une suite de variations dont il constitue le matériau thématique originel. En passant par une comparaison linguistique, on pourrait dire que la Tentation présuppose comme une sorte de grammaire transformationnelle de l'hallucination et du rêve dont l'élément premier devrait être cherché dans les souvenirs ou dans la frustration des appétits, voire, si l'on passe par Maury, dans l'effet des censures qui s'exercent à l'état de veille. Flaubert trouve donc dans l'aliénisme esquirolien ou post-esquirolien un cadre épistémique qu'il transforme en disposition rhétorique. Par le truchement d'un discours monologique, il crée d'abord une biographie lacunaire que les hallucinations sont censées compléter. Mais il va de soi que ce complément n'est pas seulement une expansion, une amplification, une transformation qui fait surgir la différence dans le même. La réduplication variée est toujours prétexte à laisser parler la copia. L'art de Flaubert, que l'on place toujours sous le signe de l'économie et de la contrainte, semble se situer, dans le déploiement des fantasmes de l'ermite, sous le signe de l'abondance, précisément parce que les tentations d'Antoine impliquent le pluriel, le sériel, l'intensif, l'hyperbolique ou, si l'on préfère, le sans fin d'un présentoir fantasmatique. Lever les censures d'Antoine par le truchement d'épisodes oniriques ou hallucinatoires, c'est aussi lever les interdits d'une esthétique placée sous le signe de l'économie, pour la situer sous celui de la dépense. Il conviendrait d'examiner comment se règle cette fête hallucinatoire, comment elle fait jouer une rythmique de l'expansion et de l'éclat; il conviendrait d'examiner également les césures qui découpent les diverses séquences ou bien encore d'étudier la manière dont celles-ci s'articulent les unes aux autres, par le jeu de glissements associatifs. On se bornera à quelques exemples.

23 La copia se thématise au sein de la section des péchés capitaux, c'est-à-dire dans la section II. Ainsi, dans la tentation de la gourmandise, les nourritures finissent par former "une "pyramide" dont les angles s'écroulent ${ }^{32}$ ». Dans la tentation de l'avarice, les pierreries s'épanchent à flots continus de manière à faire « un "monticule" sur le sable ${ }^{33}$ ». Dans la vision d'Alexandrie, reliée à la tentation de la colère, "des monuments d'architecture différente se tassent les uns près des autres ${ }^{34} »$. Dans un passage qui assure la transition entre la tentation de la colère et celle de l'orgueil, les navires sont " "tassés" dans un golfe ayant la forme d'un croissant ${ }^{35}$ ». Ce sémantisme de l'excès (" pyramide », "monticule", «tas ») est directement associé à l'aspect hyperbolique des descriptions, aux effets de liste qui parfois les structurent, à l'empilement phrastique, à la vie débordante qui est prêtée aux objets entassés : ainsi dans la tentation de la gourmandise, « les vins se mettent à couler, les poissons à palpiter, le sang dans les plats bouillonne ${ }^{36}$ ". Les choses semblent vouloir déborder de leurs limites. On retient surtout que les expressions " pyramide ", "monticule », « tas " assurent le lien d'un péché capital à un autre, par-delà les retours à la conscience claire.

Les procédures d'enchaînement sont parfois encore plus complexes que pourraient le laisser croire ces glissements thématiques. On vient de signaler que l'on passait de la tentation de la colère à celle de l'orgueil grâce à un épisode ayant valeur de suture : " Antoine monte jusqu'en haut du phare d'Alexandrie. Un grand miroir de cuivre, tourné vers la haute mer, reflète les navires qui sont au large ${ }^{37}$. " S'amorce immédiatement une 
autre séquence qui nous transporte à Constantinople où l'ermite rencontre l'empereur. Qui ne le voit, le miroir est une machine à voyager, à créer des transferts, et il en est de même des bateaux. La contiguïté de la colère et de l'orgueil s'affirme par un dispositif particulièrement habile : le miroir semble refléter par avance la séquence suivante, tout au moins il fait office d'objet transitif et associatif. Il autorise une ellipse et un passage. Il catalyse un glissement et un montage.

Dans une perspective différente, on pourrait dire que la copia fait jouer une articulation particulière de la suite et de la fin. Tous les empilements sont menacés d'effondrement, les « angles des pyramides ", on l'a vu, « s'écroulent », le monticule de pierreries a le sable pour assise, comme si la tentation était par avance condamnée, dans son épanouissement même, à s'effondrer. Mais on pourrait aussi estimer que chaque vision contient en ellemême une « suite » : «Autour du Grand-Port (Alexandrie), c'est une suite ininterrompue de constructions royales ${ }^{38}$ ». Dans le palais de Nabuchodonosor, ce sont «des superpositions d'escaliers, des suites d'arcades, des colosses, des tours ${ }^{39} »$. Tous les péchés capitaux se composent de suites, se font suite, jouent du successif, du sériel, naissent en quelque sorte d'une continuelle dérive qu'emblématise la dérive initiale et métamorphique de la paresse ; la natte « se rembourre, elle se hausse, elle devient un lit, le lit, une chaloupe; de l'eau clapote contre ses flancs ${ }^{40}$ ». L'imaginaire est sans fin, il est sans fond, comme le désir.

Qu'en conclure? Avons-nous, dans ces jeux associatifs abandonné l'univers des aliénistes post-esquiroliens ou celui de Maury? Rien n'est moins certain. Rappelons que pour Maury, dans les rêves, « les images se soudent les unes aux autres comme les diverses parties d'un panorama mouvant ${ }^{41} »$. N'oublions pas surtout que le jeu des associations se produit par la contiguïté des signifiants. Ainsi l'historien rêve de "pèlerinage » après avoir rendu visite au chimiste "Pelletier », lequel lui a donné une "pelle» de zinc ${ }^{42}$ ! Et pourtant Flaubert invente, et il invente littérairement. Il crée, en effet, une prose poétique, fondée sur des glissements, des associations, des suites, des allitérations sémantiques, tout en faisant un pas dans la direction de Freud. Car ce glissement par associations de thèmes, par un jeu sur les signifiants, cette continuité souterraine des tentations qui débordent sans cesse au-delà de la disposition censée informer la copia, cette idée que les tentations se tiennent toutes, la sexualisation implicite ou explicite des visions, nous renvoient, en effet, au-delà de l'aliénisme esquirolien, vers une autre épistémè ou plutôt nous font apercevoir dans la disposition informée par Maury et par la psychiatrie esquirolienne, une conception freudienne de la libido. Ce qui se configure, dans la séquence des sept péchés capitaux, c'est la course du désir d'un objet à un autre, c'est le désir de régression, c'est encore l'affirmation narcissique de soi. Par une progression évidente, Antoine rêve d'être reconnu comme roi : «Constantin lui pose son diadème sur le front $\left.{ }^{43} »\right)$, de devenir roi («Antoine lit, de loin, sur son front, toutes ses pensées. Elles les pénètrent, - et il devient Nabuchodonosor ${ }^{44} »$. Les hallucinations, comme le fantasme freudien, sont les trompe-la-faim momentanés d'une pulsion qui se déplace sans cesse et qui effondre néanmoins, dans la course sans fin du désir, les satisfactions fantasmatiques qu'elle se donne ${ }^{45}$.

On risquera une hypothèse supplémentaire. L'ordre dans lequel sont présentés les sept péchés capitaux ne correspond pas à celui de la catéchèse. Flaubert invente une logique nouvelle qui fait peut-être sens si l'on prend en compte, une fois de plus, l'aspect protofreudien de La Tentation. On peut s'interroger sur la suite formée par la paresse, la gourmandise, l'avarice, la colère, l'orgueil, l'envie, la luxure. Voyons de plus près les 
images qui leur sont associées. Dans la paresse, on l'a vu, la natte devient lit, et le lit une barque qui dérive sur les eaux clapotantes d'un canal. Barque mortuaire, liquide amniotique ? Flaubert évoque tout à la fois le commencement et la fin. Il énonce le désir régressif de l'ermite et la symbiose première dans le corps maternel, le souhait d'involution et la paix fœtale. La deuxième étape, centrée sur la gourmandise développe cette image célèbre : « la pulpe des fruits s'avance comme des lèvres amoureuses ${ }^{46}$. » C'est dire en même temps la succion et le sein, le baiser et la nourriture, l'indistinction fantasmée de l'être désirant et de l'objet désiré, du désir et de sa satisfaction, tout en faisant de l'oralité l'élément évident d'une libido. Troisième étape, l'avarice : Antoine veut "garder" tout, se faire "creuser dans le roc une chambre", "sentir les piles d'or s'enfoncer sous ses talons [...] » «Je veux m'en frotter le visage, me coucher dessus $!^{47}$ », déclare-t-il. Faut-il gloser sur la valeur excrémentielle de l'or, sur ce barbouillage puéril, sur cette rétention dans une crypte? On ne s'attardera pas davantage sur les meurtres qui, dans la tentation suivante, emblématisent la colère et qui semblent le volet sadique du stade sadique-anal. Tout se passe donc comme si les péchés capitaux, dans la disposition adoptée par Flaubert, se succédaient conformément aux analyses de Freud sur les stades du développement psychique de l'enfant. Mais il semble, toutefois, que la machine s'enraye. Certes, on serait tenté de penser qu'Antoine, qui se fait sacrer par Constantin, qui se substitue à Nabuchodonosor, prend ainsi la place du père. Stade œdipien? Point de femme dans cet épisode. Certes, la reine de Saba va surgir, mais sans que sa venue s'accorde avec la séquence précédente. Tout se passe comme si les scénarios œdipiens demeuraient inachevés, incomplets, alors que s'impose nous l'avons vu, le narcissisme de l'ermite sous la forme d'un moi-roi. Mais précisément, ce qui caractérise la vie érémitique n'est-ce pas un défaut d'autrui ? Dans les tentations, l'autre est un leurre. Le texte flaubertien repose sur un altruicide premier qui débouche à terme sur la mise en cause de ce qui est l'Autre absolu, le logos ordonnateur du monde.

On déplacera notre propos et nos interrogations, en se souvenant des accusations portées contre Antoine par son disciple Hilarion, c'est-à-dire par son double dont on a vu qu'il semblait le fruit d'une hallucination psychique :

Hypocrite qui s'enfonce dans ta solitude pour se livrer mieux au débordement de ses convoitises! Tu te prives de viandes, de vin, d'étuves, d'esclaves et d'honneurs ; mais comme tu laisses ton imagination t'offrir des banquets, des parfums, des femmes nues et des foules applaudissantes! Ta chasteté n'est qu'une corruption plus subtile, et ce mépris du monde l'impuissance de ta haine contre lui ! C'est là ce qui rend tes pareils si lugubres, ou peut-être parce qu'ils doutent. La possession de la vérité donne la joie. Est-ce que Jésus était triste ${ }^{48}$ ?

Rapprochons ce discours d'un passage de l'Éthique:

Seule assurément, une farouche superstition interdit de prendre des plaisirs. En quoi, en effet, convient-il mieux d'apaiser la faim et la soif que de chasser la mélancolie? Telle est ma règle, telle est ma conviction. Aucune divinité, nul autre qu'un envieux, ne prend plaisir à mon impuissance et à ma peine, nul autre ne tient pour vertu nos larmes, nos sanglots, notre crainte et autres marques d'impuissance intérieure ; au contraire, plus grande est la Joie dont nous sommes affectés, plus grande la perfection à laquelle nous passons, plus il est nécessaire que nous participions de la nature divine. Il est d'un homme sage, dis-je, d'user des choses et d'y prendre plaisir autant qu'on peut (sans toutefois aller au dégoût, ce qui n'est plus prendre plaisir ${ }^{49}$ ).

30 On ne saurait critiquer plus sévèrement l'ascétisme et faire de l'incarnation le principe de la joie, celle-ci étant agrandissement du territoire de l'homme, ou tout moins signe de 
son épanouissement moral. Dans le dialogue imaginaire avec soi, qui renvoie au fractionnement de la personnalité tel que le concevaient Maury et Baillarger, Flaubert fait surgir en tiers l'éthique spinozienne. Un dialogue philosophique s'insinue dans le cadre rhétorique induit par une disposition psychologique, celle d'un sujet qui, dans le rêve ou sous l'effet d'une hallucination, argumente contre lui-même. On le voit, nous sommes passés d'un savoir à un autre, de Maury à Freud, pour aller ensuite vers Spinoza et peutêtre vers Nietzsche, car, dans sa mise en cause de la tristesse, du pénitentiel, du sacrificiel, Flaubert assure une transition, un lien, entre les deux philosophes.

31 Que voulions-nous montrer dans cette navigation au sein des péchés capitaux ? Certes que l'art d'un écrivain consiste à transformer les données épistémiques en cadre rhétorique ; certes que le texte de la Tentation semble s'approcher du freudisme, peut-être au nom de ce que l'inventeur de la psychanalyse appelait la connaissance endopsychique de l'écrivain. Il nous a plu de souligner que c'est en créant une forme, en cherchant à régler la copia, à lui donner un rythme, en suscitant des récurrences et des enchaînements qui signifient (mais pas seulement sur le plan rhétorique) que Flaubert faisait penser la fiction. On n'instaure pas pour autant le freudisme en point ultime d'une psychologie ; on le croit daté, provisoire comme tout savoir en sciences humaines. On voulait également suggérer, et voilà qui va nous faire revenir à Brierre de Boismont, qu'il ne convenait pas d'assimiler trop rapidement Flaubert peinant sur son travail au saint halluciné sous l'effet de son ascétisme. «L'ermite de 1874 identifie dans ses hallucinations les auteurs anciens utilisés ou produit des nouvelles histoires à partir des épisodes de la Bible. N'est-il pas une image de l'artiste en fin de métamorphose ${ }^{50}$ ", se demandait Gisèle Séginger. Nous pensons, tout au contraire, que le texte de La Tentation joue d'une similitude et d'une dissimilitude. Il contraint le lecteur à instaurer un rapprochement analogique entre Antoine et le metteur en scène des Tentations, mais il maintient toujours entre eux une distance, de manière à différencier l'imagination créatrice de l'écrivain et l'imagination hallucinée de l'ermite. Les deux ne se confondent pas, ne se superposent pas.

Certes, Antoine est inventeur de fictions, mais involontairement. Les images s'enchaînent, dans la psyché de l'ermite, pas le biais de signifiants, par des glissements langagiers, par des associations thématiques et métaphoriques; cette combinatoire n'est pas le fait de l'ermite, mais de Flaubert qui recrée, à partir de ses lectures et de sa propre expérience des hallucinations, la vie intérieure de son personnage. L'un n'est pas la projection de l'autre. Tout se passe en réalité comme si l'écrivain prenait appui sur la psychiatrie de son temps pour inventer les fantasmes de son personnage, tout en se dirigeant en même temps vers une autre rive. Il se fonde sur Brierre de Boismont et sur la distinction que celui-ci opérait entre hallucination physiologique et hallucination pathologique pour se soustraire, dans sa correspondance, aux attendus de Taine en contournant à la fois le questionnaire que celui-ci lui soumet et en infléchissant, parallèlement, dans un sens qui lui est propre, les souvenirs de ses lectures psychiatriques.

Du reste, écrivait-il à Taine, n'assimilez pas la vision intérieure de l'artiste à celle de l'homme vraiment halluciné. Je connais parfaitement les deux états : il y a un abîme entre eux. - Dans l'hallucination proprement dite, il y a toujours terreur, on sent que votre personnalité vous échappe, on croit qu'on va mourir. Dans la vision poétique, au contraire, il y a joie. C'est quelque chose qui entre en vous. - Il n'en est pas moins vrai qu'on ne sait plus où l'on en est ${ }^{51}$ ?

Cette dichotomie entre deux états est assez forte pour que l'on ne puisse glisser d'un type d'hallucination à une autre, même si dans l'hallucination créatrice, au même titre que ce qui se joue dans l'hallucination pathologique, « l'image imaginée, [je rectifie le texte de la 
Pléiade en prenant appui sur une lecture proposée par Sandra Janssen ${ }^{52}$, semble aussi vraie que la réalité objective des choses ${ }^{53}$.»

Le romancier déjoue encore le psychologue interrogateur en faisant apparaitre que les deux états diffèrent non seulement à cause des affects qui leur sont associés, mais aussi en raison de la relation qu'ils entretiennent avec le réel. L'hallucination pathologique, signale Flaubert, s'ajoute à la réalité sans l'annuler, en revanche l'hallucination artistique ne s'emboîte pas dans « la réalité ambiante ». «Je ne sais plus ce qu'il y a autour de moi. J'appartiens à cette apparition, exclusivement ${ }^{54} "$, déclare l'écrivain.

Si le clivage entre ces deux formes d'hallucination répercute, comme on vient de le voir, les thèses des aliénistes de la Monarchie de Juillet, l'écrivain les distord cependant dans un sens que nous allons préciser. Certes, en bon esquirolien, Flaubert voit dans l'hallucination pathologique, comme il l'écrit à Taine, « une maladie de la mémoire ", « un feu d'artifice de réminiscences ${ }^{55} »$. Tout comme Brierre, il postule que l'hallucination artistique, sous l'effet de l'attention, annulerait le monde extérieur qui entoure l'artiste. L'image s'imposerait alors à la fois comme une réalité négative et comme une sensation, elle aurait l'évidence d'une perception. Imaginer, ce serait donc être absolument présent aux images que l'on crée sous l'effet d'une concentration de l'esprit. Dans le prolongement des débats qui se sont déroulés en 1855-1856, à la Société médicopsychologique, Flaubert incite même Taine à demander «à des musiciens s'ils "entendent" absolument la musique qu'ils vont écrire, comme nous les romanciers, nous voyons nos bonhommes ${ }^{56} »$.Toutefois, même si l'écrivain semble accréditer, dans un premier temps, la relation entre hallucination artistique et idéalisation du réel («Ce que la réalité m'a fourni, au bout de très peu de temps, ne se distingue plus pour moi des embellissements ou modifications que je lui ai donnés $\left.{ }^{57} »\right)$, s'il adopte, semble-t-il, les postulats de Brierre sur l'écoute intérieure, la représentation intérieure, l'hallucination artistique, telle qu'il la conçoit, ne projette pas sous forme de sensation-image un déjà-là. Les formes qu'elle suscite sont absentes de toute réalité ; pour le dire en d'autres termes, elles sont des articles de pure fiction. Et en cela, nous le voyons, nous délaissons Brierre, pour aller à la rencontre de Mallarmé. L'hallucination artistique a besoin du tremplin du négatif pour imposer ses visions. Elle existe comme une sorte d'îlot psychique sans corrélat :

Dans l'hallucination artistique, le tableau n'est pas bien limité, quelque précis qu'il soit. Ainsi, je vois parfaitement un meuble, une figure, un coin de paysage, mais cela flotte, cela est suspendu, ça se trouve je ne sais où. Ça existe seul et sans rapport avec le reste ${ }^{58}[\ldots]$

C'est bien parce qu'elle se présente comme un isolat que l'hallucination artistique est déjà création ; elle n'est engânée ni dans le réel, ni dans une biographie, elle existe déjà per se, elle est déjà littérature. Tout au moins le discours que Flaubert développe à son propos est entièrement tributaire non point tant peut-être d'une réalité vécue que de l'idée qu'il se fait de la création littéraire et de sa relative autonomie.

On ne cherchera pas à conclure, sinon en revenant sur des questions de méthode. Parce qu'il fallait passer constamment d'un champ à un autre, d'un débat que l'on dira psychologique à des questions d'esthétique, d'un dialogue entre aliénistes à un dialogue entre un écrivain et un essayiste psychologue, nous avons été contraint à tracer des méandres, à faire des boucles. La ligne droite fut notre ennemie. Toujours dans ces échanges, il nous importait de faire apparaitre le jeu des différences, des variations, des tensions. Plutôt que de délimiter la cartographie d'une épistémè, notre ambition fut de 
saisir des inflexions. On rêve, et ce parcours en témoigne, d'une lecture qui ne se préoccuperait pas de trouver du même, que celui-ci soit thématique, sociologique, structural, mais qui considérerait, à partir de motifs carrefours (ici les hallucinations), la nature différentielle des échanges, des discours. L'histoire des idées ne devrait-elle pas être l'histoire des différences? C'est aussi au nom de ce principe que nous nous sommes efforcé de montrer, à propos de Flaubert, comment la mise en scène d'un savoir médical conduisait insensiblement vers un autre savoir. La littérature extrait du déjà-su ce qu'elle-même ne sait pas encore. L'opérateur de ce glissement ou de cette ouverture, c'est le travail sur une forme-sens, par où s'affirme la spécificité, parmi tous les autres discours, des écrits littéraires. Ils inventent sur le plan rhétorique et ils éprouvent leur pensivité dans la reformulation d'un déjà-dit, auxquels ils semblent pourtant souscrire.

\section{NOTES}

1. Jean-Étienne-Dominique Esquirol, Des maladies mentales considérées sous le rapport hygiénique et médico-légal, Baillière, t. I, 1838, p. 192.

2. Voir Jules Baillarger, «Des hallucinations, des causes qui les produisent, des maladies qu'elles caractérisent ", Mémoire de l'Académie de médecine, t. XII, p. 488.

3. Louis Peisse, Annales médico-psychologiques, 1856, II, p. 282.

4. Alexandre Brierre de Boismont, Des hallucinations ou histoire raisonnée des apparitions, des visions,

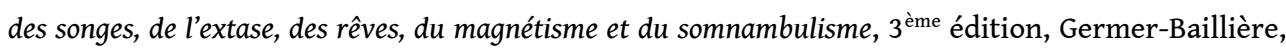
1862, p. 466. Ibid., p. 300-301.

5. Alfred Maury, Le Sommeil et les rêves [1861], Didier, 1865, p. 432.

6. Voir Tony James, Vies secondes, Gallimard, "Connaissance de l'inconscient », 1995 et Juan Rigoli, Lire le délire, Fayard, 200.

7. Louis Peisse, Annales médico-psychologiques, 1856, II, pp. 286-287.

8. Hippolyte Taine, De l'intelligence, Hachette, t. I, 1870, p. 413.

9. Louis Francisque Lélut, "De l'amulette de Pascal », communication lue à l'Académie des sciences morales et politiques, séance du 14 et du 21 décembre 1844, Annales médico-psychologiques , V, 1, 1845.

10. Alexandre Brierre de Boismont, Des hallucinations..., éd. cit., p. 466.

11. Moreau cite et commente Le Rêve et la vie (entendons Aurélia) : "Gérard de Nerval n'était point un aliéniste, mais, malheureusement pour lui, il avait été aliéné, et pour parler de la folie, il n'avait qu'à puiser dans son for intérieur : il lui suffisait de raconter ses impressions intimes, sans idées préconçues, et surtout sans vouloir faire de la science: ses paroles devaient donc reproduire au vif, et avec une rigueur absolue, l'état de son âme pendant son délire. Or, nous venons de voir que pour peindre cet état, il ne trouve d'autre expression que celle-ci: "l'épanchement du songe dans la vie réelle".

Après Gérard de Nerval, qu'on me pardonne de parler un peu de moi-même. Je suis aliéniste, et, de plus, m'étant volontairement plongé dans un état de folie artificielle (folie identique à la folie spontanée, du moins au point de vue des phénomènes physiologiques), j'ai pu me prendre moimême pour sujet de mon observation. Alors pour moi la lumière s'est faite dans les ténèbres [...] Il me fut démontré, dès lors, et ma conviction est la même aujourd'hui que la folie n'était, en effet, comme Gérard en eut la pensée, que “l'épanchement du songe dans la vie réelle”. C'est 
précisément ce que j'avais dit dans les mêmes termes (voir notre livre du haschisch). » (La Psychologie morbide...., Victor Masson, 1859, pp. 430.)

Juan Rigoli a commenté cette page et nous renvoyons à son beau livre, op. cit., p. 552-570. Nous importe ici l'effet circulaire qui garantit la vérité littéraire sur l'expérience de l'aliéniste et le savoir de celui-ci sur la littérature nervalienne. Nous importe encore la réduction en quelque sorte monosémique du discours nervalien qui est pourtant bifide. D'une part, celui-ci, dans les premières pages d'Aurélia semble mimer le discours médical, d'autre part, il n'a de cesse de lui échapper, en transformant en savoir et en initiation sa plongée dans le rêve.

12. Alexandre Brierre de Boismont, op. cit., p. 256.

13. Ibid., p. 22. L'aliéniste invoque une "disposition de l'âme que tout le monde a éprouvée », la rêverie, dans laquelle il aperçoit des phénomènes parfois proches de l'hallucination. Il se réfère ensuite à une lettre de Vigny qui suggère que la rêverie peut être dangereuse pour les âmes faibles, mais qu'elle constitue pour les «forts" «la source des grandes choses et des folles entreprises ». Cette lettre de Vigny, telle que Brierre la présente, semble annoncer l'admirable Promontorium somnii hugolien.

14. Voir Brierre, op. cit., p. 507. Les grands hommes sont « les messagers de l'infini ». Ceux qui agissent dans l'histoire, et Jeanne d'Arc peut être classée parmi eux, connaissent des extases de savoir, ils sont parfois visités par des apparitions qui sont autant de manifestations des hallucinations physiologiques.

15. Ibid., p. VI. Brierre se rapporte à un article de Victor Cousin, «La jeunesse de Madame de Longueville ", Revue des Deux Mondes, 15 mai 1852, p. 625.

16. Brierre cite l'article paru dans L'Artiste le 13 mars 1858. Voir Brierre, op. cit., p. 462.

17. Brierre (op. cit., p. 464) se réfère au grand article sur Balzac, paru dans le Journal des débats.

18. Brierre, op. cit., p. 464.

19. Ibid., p. 465.

20. Alfred Maury, op. cit., p. 91.

21. Ibid., p. 51.

22. Ibid., p. 120.

23. G. Flaubert, La Tentation de saint Antoine, Flammarion, «G-F », 1967, p. 34. Précisons qu'en racontant son apprentissage auprès de Didyme, Antoine rappelle qu'il coudoyait sur le port des "gymnosophistes du Gange frottés de bouse de vache» (p. 33), des «sectateurs de Manès, de Valentin, de Basilide, d'Arius [...]» (p. 34)

24. Ibid., p. 50.

25. Ibid., p. 36-37.

26. Ibid., p. 39-40.

27. « On pourrait donc modifier ce qui paraît être de l'ordre de l'immuable ? (Ibid., p. 40).

28. Ibid., p. $35:$ : [...] il m'adressait à chaque instant des questions ».

29. Ibid., p. 75.

30. Ibid., p. 43.

31. Ibid., p. 39.

32. Ibid., p. 51.

33. Ibid., p. 53.

34. Ibid., p. 54.

35. Ibid., p. 57.

36. Ibid., p. 51.

37. Ibid., p. 57.

38. Ibid., p. 55.

39. Ibid., p. 59.

40. Ibid., p. 49.

41. Alfred Maury, op. cit., p. 117. 
42. Ibid., p. 115.

43. G. Flaubert, La Tentation de saint Antoine, éd. cit., p. 59.

44. Ibid., p. 60.

45. Sur cette course du désir, voir Jeanne Bem, Désir et savoir dans l'œuvre de Flaubert, Neufchatel, À la Baconnière, 1979, p. 91.

46. G. Flaubert, La Tentation de saint Antoine, éd. cit., p. 51.

47. Ibid., p. 53.

48. Ibid., p. 78.

49. Baruch Spinoza, Éthique, traduction par Charles Appuhn, IV e partie, proposition XLV.

50. Gisèle Séginger, Naissance et métamorphose de l'écrivain, Champion, 1997, p. 44.

51. G. Flaubert, lettre à Hippolyte Taine, novembre 1866, Correspondance, édition établie par Jean Bruneau, Gallimard, « Bibliothèque de la Pléiade », t. III, 1991, p., 562.

52. Voir Sandra Janssen, Une archéologie des conceptions de l'imagination en psychologie et littérature, 1840-1930 (Flaubert, Tchekhov, Musil), thèse soutenue en 2006 à l'Université libre de Berlin.

53. Gustave Flaubert, lettre à Hippolyte Taine [novembre 1866], Correspondance, éd. cit., t. III, p. 562 .

54. Gustave Flaubert, lettre à Hippolyte Taine [1 $\mathrm{1}^{\mathrm{er}}$ décembre 1866], Correspondance, éd. cit., t. III, p. 573.

55. Ibid.

56. Ibid.

57. Gustave Flaubert, lettre à Hippolyte Taine [novembre 1866], Correspondance, éd. cit., t. III, p. 562.

58. Gustave Flaubert, lettre à Hippolyte Taine [novembre 1866], Correspondance, éd. cit. t. III. P. 573.

\section{RÉSUMÉS}

A la suite d'Esquirol dans son traité Des maladies mentales de 1838, ceux que l'on appelait alors les "aliénistes» proposent de nouvelles théories de l'imagination qui, considérées dans leur ensemble, esquissent un cadre épistémique que Flaubert utilise comme cadre rhétorique dans la troisième version de la Tentation de saint-Antoine. Toutefois, l'auteur n'accepte pas l'assimilation de l'état dans lequel se trouve l'artiste lorsqu'il crée à une hallucination pathologique. Il s'oppose en cela à la théorie de Moreau de Tours; en revanche, il se montre plus proche de la pensée de Brierre de Boismont (et de celle de l'historien Alfred Maury). Par cet usage intelligemment mitigé du cadre épistémique de l'aliénisme contemporain et par l'invention d'une prose poétique dont les soubassements théoriques annoncent certaines conceptions de Freud et de Nietzsche, Flaubert dépasse résolument les esquiroliens.

\section{INDEX}

Index chronologique : XIXe siècle

Mots-clés : aliénisme, Gustave Flaubert, Brierre de Boismont, hallucination, mémoire, imagination, rêve, vision, création artistique, psychologie 


\section{AUTEUR}

\section{JEAN-LOUIS CABANÈS}

Université Paris Ouest Nanterre la Défense 